\title{
Lung Cancer Screening: The Radiologist's Perspective
}

\author{
Mathias Prokop, MD, $\mathrm{PhD}^{1}$ \\ ${ }^{1}$ Department of Radiology, Radboud University Medical Center, \\ Nijmegen, The Netherlands \\ Semin Respir Crit Care Med 2014;35:91-98.
}

\begin{abstract}
Address for correspondence Mathias Prokop, MD, PhD, Department of Radiology, Radboud University Medical Center, Nijmegen, The Netherlands (e-mail: Mathias.Prokop@radboudumc.nl).
\end{abstract}

\begin{abstract}
\section{Keywords}

- lung cancer screening

- low-dose CT scans

- pulmonary nodules

Lung cancer is the leading cause of cancer death worldwide and accounts for more deaths than breast, prostate, colon, and pancreatic cancers combined. A distinct minority (15\%) of lung cancers are diagnosed at an early stage; 5-year survival (all lung cancers) approximates 15\%. Randomized, controlled trials in the 1960s and 1970s found that chest radiographic screening did not confer a survival benefit for high-risk patients. Recently, however, the randomized, controlled National Lung Screening Trail (NLST) provided category 1 evidence that low-dose computed tomography (CT) screening conferred a significant survival benefit for screened individuals: lung cancer-specific mortality was reduced by $20 \%$ after 6.5 years of follow-up; even all-cause mortality decreased by $6 \%$. The positive outcome triggered many national medical societies in the United States to recommend lung cancer screening in high-risk individuals. However, the favorable results of the NLST trial have not yet been reproduced. Currently, nine randomized, controlled trials are being or have been performed in various European countries. In contrast to the NLST study, three published European studies found no benefit from low-dose CT scanning in at-risk patients. Additional studies are required to establish the benefit and risks associated with repetitive low-dose CT for screening at-risk patients. Many unanswered questions remain. Who should be screened and how often? What is the appropriate workup when lesions are noted in asymptomatic individuals? What is the risk of cumulative radiation exposure from repetitive low-dose CT scans? What is the responsibility of health care personnel to evaluate nonpulmonary issues detected by CT (e.g., coronary calcifications). In this review, we address these and other questions that arise. Further, implementation of screening programs may be logistically difficult, require additional personnel and computer software, and will incur significant health care costs.
\end{abstract}

Lung cancer is the leading cause of cancer death worldwide and accounts for more deaths than breast, prostate, colon, and pancreatic cancer combined. The 5-year survival rates are around 15\%, compared with between 64 and 99\% for colon, breast, and prostate cancer. A minority of $15 \%$ of cases are diagnosed at an early stage. ${ }^{1}$ Lung cancer incidence increases with age and duration of smoking as well as a variety of other risk factors. The Lung Cancer Mortality Reduction Act, passed by the US Congress in 2011, summarizes in its findings that, because of the strong association with smoking, lung cancer is the only cancer blamed on patients despite the fact that some $60 \%$ of patients are former smokers and 20\% have never smoked. $^{2}$ It also estimates the value of life lost at more than $\$ 433,000,000,000$ per year by 2020 and states that early detection might save more than 70,000 lives per year in the United States.
Issue Theme Thoracic Imaging; Guest Editor, Martine Remy-Jardin, MD, PhD
Copyright (c) 2014 by Thieme Medical Publishers, Inc., 333 Seventh Avenue, New York, NY 10001, USA. Tel: +1(212) 584-4662.
DOI http://dx.doi.org/ 10.1055/s-0033-1363455. ISSN $1069-3424$. 


\section{Why Computed Tomography Lung Cancer Screening?}

Lung cancer carries a substantially increased chance of survival if detected at an early stage, with close to $100 \%$ survival for a minimally invasive adenocarcinoma (MIA). ${ }^{3}$ Already in the 1960s and 1970s, efforts had been undertaken to detect lung cancer early by using chest radiography screening. All randomized, controlled trials, however, did not show a survival benefit for those screened despite the fact that more cancers were detected. ${ }^{4}$ In fact, recently, the PLCO trial reconfirmed these early finding: screening with chest radiography did not show a significantly increased survival. ${ }^{5}$ Other efforts using serum markers, genetic testing, or analysis of exhaled gas were not successful in being able to detect cancer at an early stage. ${ }^{6}$

Recently, however, the randomized, controlled National Lung Screening Trail (NLST) provided category 1 evidence that low-dose computed tomography (CT) screening is able to provide a significant survival benefit for screened individuals: lung cancer-specific mortality was reduced by 20\% (95\% confidence interval, 6.8-26.7) with the 6.5 years of followup; even all-cause mortality decreased by $6 \%{ }^{7}$ Because of the failure of previous screening attempts, these results are truly spectacular and contradicted previous predictions made from model calculations: these model calculation predicted 3.2 times increase in number of cancers but no benefit in survival or advanced cancer stages. ${ }^{8}$ The NLST provided evidence to the contrary.

The positive outcome and the ensuing evaluation of the results of the NLST triggered many national medical societies in the United States to recommend lung cancer screening in high-risk individuals. Among those who recommend screening are the American Association for Thoracic Surgery (AATS), the American College of Chest Physicians, the American Society of Clinical Oncology, and the American Cancer Society.
Recently, the National Comprehensive Cancer Network (NCCN) lung cancer screening panel provided a positive advice.

However, although the positive recommendations are being issued, several aspects remain to be addressed: since the NLST is not the only randomized controlled on low-dose CT screening, it remains to be seen whether the positive results of NLST can be reproduced. Once screening is being implemented, the question is who should be screened and for which period of time? Should screening be offered as a onetime exam or only within a screening program? How should the process of screening be organized and what should be the procedure once a lung lesion is found? What should be the workup of a lesion that is deemed suspicious? How can screening-detected small cancers best be treated? How can we manage the expectations of those screened? And finally, should screening for lung cancer be extended to chest screening, in which other common diseases visible on noncontrastenhanced low-dose chest CT are also evaluated? This article tries to provide an overview of current knowledge about these questions.

\section{Can Results Be Reproduced by Other Large Trials?}

In Europe, currently eight randomized, controlled trials on low-dose CT for lung cancer screening are being or have been performed in various countries: NELSON in the Netherlands and Belgium; Danish Lung Cancer Screening Trial (DLCST) in Denmark; DANTE, MILD, and ITALUNG in Italy; LUSI in Germany; Depicscan in France; and UKLS in the United Kingdom. So far, the three published trials do not support the NLST results (- Table 1): the DLCST showed increased allcause mortality in the CT screening $\mathrm{arm}^{9}$; the Italian DANTE trial showed no effect. ${ }^{10}$ The Italian MILD trial showed no effect of 2-yearly screening compared with no screening and a

Table 1 Mortality due to lung cancer and other causes reported by NLST and three European trials,9-11

\begin{tabular}{|c|c|c|c|c|c|}
\hline & Follow-up (N) & Mortality (mo) & All cause & Cancer & Other \\
\hline \multicolumn{6}{|l|}{ NLST } \\
\hline LDCT & 26,722 & 78 & 1,877 & 356 & 1,521 \\
\hline CXR & 26,732 & 78 & 2,000 & 443 & 1,557 \\
\hline \multicolumn{6}{|l|}{ DLCST } \\
\hline LDCT & 2,052 & 58 & 61 & 15 & 46 \\
\hline Controls & 2,058 & 58 & 42 & 11 & 31 \\
\hline \multicolumn{6}{|l|}{ DANTE } \\
\hline LDCT & 1,276 & 34 & 46 & 20 & 26 \\
\hline Controls & 1,196 & 34 & 45 & 20 & 25 \\
\hline \multicolumn{6}{|l|}{ MILD } \\
\hline $\mathrm{LDCT} / \mathrm{a}$ & 1,190 & 54 & 31 & 12 & 19 \\
\hline $\mathrm{LDCT} / 2 \mathrm{a}$ & 1,186 & 54 & 20 & 6 & 14 \\
\hline Controls & 1,723 & 54 & 20 & 7 & 13 \\
\hline
\end{tabular}

Note: The MILD trial compared annual and biannual screening with no screening. 
negative effect of yearly screening with more detected cancers but also higher all-cause mortality. ${ }^{11}$ The largest European trial, NELSON, has not yet been published. Publication is expected in 2015 to 2016.

All published European trials, except possibly NELSON, are not sufficiently powered to be able to demonstrate a significant mortality benefit. However, instead of demonstrating no difference between screening and no screening, some European trials suggest a negative effect of screening. This is more disconcerting as the results cannot be explained well. Potential reasons include relatively small sample sizes, inclusion of a lower risk screening population but also more complications from the workup and treatment of patients in the screening arm. By the time NELSON data can be pooled with the other European data, the sample size should be large enough to enable meaningful comparison with NLST. The outcome of the analysis of these pooled data might confirm NLST, but there is a realistic chance that the data might also contradict NLST. Caution is therefore warranted.

\section{Who Should Be Screened?}

When selecting the most appropriate target group for screening, screening of high-risk individuals will yield higher detection rates and should therefore increase benefits relative to the risks resulting from radiation and false-positive results. The cancer detection rates during the first round of screening varied between $0.8 \%$ in the DLCST and 3.7\% in the DANTE trial. ${ }^{9,10}$ Both had $\geq 20$ pack-years as inclusion criteria, but the age range varied between 50 and 70 years for DLCST and 60 to 74 years for DANTE. For comparison, the NLST with $\geq 30$ pack-years and an age range of 55 to 74 years yielded a 1.0\% baseline cancer detection rate. ${ }^{7}$ The cancer detection rate in all published trials varies less during follow-up and lies between 0.6 and $0.8 \%{ }^{7-11}$ There are various computational models for prediction of lung cancer risk that take into account age, sex, active and passive smoking history, history of pneumonia, sociodemographic factors, and exposure to pulmonary toxic substances. ${ }^{6}$ These models yield a good predictive power for individual risk prediction and have the potential to be superior to simple age and smoking history criteria used in most current trials. At present, efforts are made to expand these models by including genetic and other biomarkers but success has been limited. ${ }^{12}$

The category 1 evidence for a 20\% reduction of lung cancer mortality in the NLST is strictly valid only for individuals that fulfill the inclusion criteria, namely, current and former smokers with 30 pack-years of smoking history and age between 55 and 74 years; the former smokers had quit smoking within past 15 years. To be reasonably sure that a screening program has a positive effect, these or even stricter inclusion criteria should be applied. Consequently, the AATS opted for a $5 \% 5$-year-risk threshold for screening, ${ }^{13}$ which is above the cancer risk seen in NLST. On the contrary, the NCCN opted for less stringent criteria, a lower age threshold of 50 years and a 20 pack-years smoking history, ${ }^{14}$ that, however, come closer to the ones used for NELSON. Without a suitable randomized, controlled trial, however, the success of these enlarged inclusion criteria on survival can only be modeled.

The issue of lung cancer in non-smokers needs to be discussed: this group encompasses $20 \%$ of the total lung cancer population and is sixth leading cause of cancer death ${ }^{2}$ but does not fit the inclusion criteria of recent screening trials. To be able to tackle this group as well, a high-risk subgroup needs to be identified based on personal characteristics, family history, and novel biomarkers including genetics. Little progress has been made in this field yet.

\section{What to Do with Screening-Detected Solid Nodules?}

Most early lung cancer screening trials, such as the Early Lung Cancer Action Project, were using single-slice CT scanners and evaluated any nodules found that did not have clearly benign characteristics. ${ }^{14}$ This was possible because the detection threshold of these early techniques was around $5 \mathrm{~mm}$. With the advent of multidetector CT in 1998, much smaller nodules could be detected routinely. This required a change of the course of action. The NLST classified any noncalcified nodule or mass $\geq 4 \mathrm{~mm}$ in diameter as a positive and therefore suspicious nodule. This resulted in some $27 \%$ of positive results during the first and second screening round of NLST. ${ }^{7}$ Suspicious nodules were sent for further evaluation, which could be, for example, a follow-up CT, positron emission tomography (PET) imaging, bronchoscopy, or biopsy. No strict guidelines were applied.

The nodule management approach chosen by NELSON, DLST, MILD, and LUSI was based on the volume of solid nodules. Volumetry is able to detect growth much more readily than diameter measurements (-Fig. 1): an increase in diameter by $25 \%$, which is in the range of the measurement error for 5 to $10 \mathrm{~mm}$ nodules, presents almost as doubling of the nodule volume. Given that the reproducibility of volume measurements is also in the range of $25 \%$, much smaller changes can be picked up. Because of substantial variation between various volumetry software tools, however, one has to ensure that identical software is used for follow-up measurements. ${ }^{15}$

The nodule management approach by NELSON distinguished benign (calcifications, fat, infection), too small to evaluate (15-50 $\mathrm{mm}^{3}$, corresponding to an average diameter of 3-4.5 mm), indeterminate (50-500 $\mathrm{mm}^{3}$; average diameter $4.5-10 \mathrm{~mm}$ ), and actionable nodules ( $>500 \mathrm{~mm}^{3}$; average diameter $>10$ $\mathrm{mm}$ ). Actionable nodules were referred to a pulmonologist for further evaluation. Indeterminate nodules were rescanned after 3 months, and no action was taken for all other nodules because participants would be entering the second screening round after 1 year anyway. On follow-up scans, the growth rate of each nodule was determined by calculating the volume doubling time. Nodules with a volume doubling time less than 400 days were considered suspicious and referred to a pulmonologist. ${ }^{16}$ Using this approach, the number of positive screening results could be reduced to $2.6 \%{ }^{17}$

Because of measurement variability, however, the determination of volume doubling times deteriorates as follow-up 

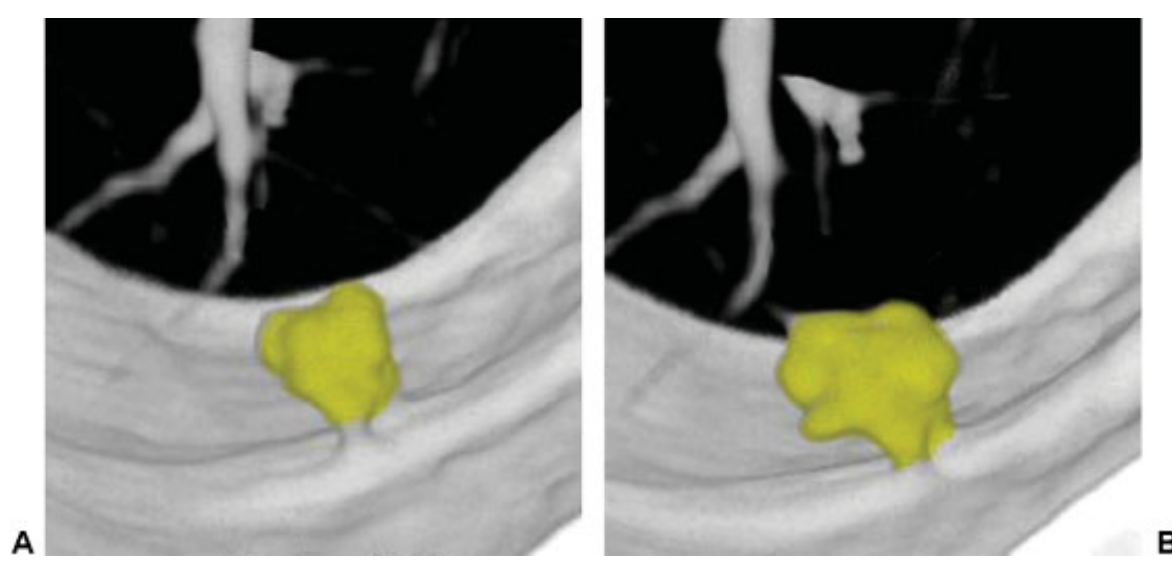

Fig. 1 (A and B) Nodule that grew from 8 to $9.6 \mathrm{~mm}$ (20\%) within 3 months. This corresponded to a much bigger increase in volume from 280 to $460 \mathrm{~mm}^{3}(64 \%)$. The growth rate corresponds to a volume doubling time of approximately 125 days, which signifies a fast-growing lesion. Histology was adenocarcinoma.

intervals shorten. At the chosen threshold of 400 days, only $14.7 \%$ of growing nodules at 3-month controls turned out to be malignant, as compared with $50 \%$ of nodules at 1 -year follow-up. ${ }^{18}$ By reducing the threshold to 230 days at 3 months, about one-third less of the participants with benign nodules were referred for further workup. ${ }^{19}$ Since measurement errors also increase with smaller nodule size, one should consider to increase the follow-up interval to 6 months for small indeterminate nodules in the range of 50 to $200 \mathrm{~mm}^{3}$ (average diameter $4.5-8 \mathrm{~mm}$ ).

In the course of the past decade, a type of nodule has been identified that has such a high likelihood of being benign that follow-up is not warranted: these so-called perifissural nodules are characterized by their proximity to the pleura or fissures. They have a specific shape (often lentiform or triangular) that allows to identify them. Histologically, they most likely represent perifissural lymph nodes, although few have been resected. Despite the fact that they may show malignant growth rates at follow-up, they will eventually shrink again and prove benign. ${ }^{20}$

Recently, the group from Vancouver published a model that estimates the risk of malignancy based on nodule characteristics on baseline screening exams. ${ }^{21}$ The main predictor is nodule size: probability of malignancy of a nonspiculated nodule increased from $0.3 \%$ for 5 -mm nodules to $1.8 \%$ for 8 $\mathrm{mm}$ nodules and $3.3 \%$ for $10-\mathrm{mm}$ nodules. Spiculation increased risk by a factor of 2.5 while female gender and upper lobe location increased it by a factor of 1.8 to 1.9. This work provides the basis for an approach to nodule follow-up based on modeling the cancer risk for individual nodules.

\section{What to Do with Screening-Detected Subsolid Nodules?}

The early stages of the cancer sequence from atypical adenomatoid hyperplasia to MIA and finally invasive adenocarcinoma present as subsolid pulmonary nodules on CT images. ${ }^{22}$ Subsolid nodules are categorized either as ground glass nodules and semisolid nodules. Ground glass nodules display a hazy increased attenuation that does not obliterate the underlying bronchial or vascular structures on thin-section $\mathrm{CT}$, whereas semisolid nodules consist of both a ground glass and a solid component of soft tissue density.

The significance of subsolid nodules within the development of lung cancer has only become apparent within the past decade based on screening studies. Although solid pulmonary nodules detected during lung cancer screening have an average cancer risk of $7 \%$, subsolid nodules have an almost five times higher risk for being malignant. ${ }^{23}$ The prevalence of solid malignancies is approximately $1 \%$ in a screening setting, the prevalence of subsolid malignancies is not yet known. Subsolid malignancies tend to grow much slower than solid ones ${ }^{22}$ and have an excellent 5 -year survival. $^{3}$

Because of their slow growth but high malignancy rate, subsolid lesions pose a growing clinical challenge. Just like early low-malignancy prostate cancer, many of these lesions will not kill the patient while a few others will progress to invasive tumors that have a much lower 5-year survival. The Fleischner society published guidelines for management of subsolid lesions taking into account their slow growth but high risk. ${ }^{22}$ In these guidelines, management is based on follow-up and the presence and growth of a solid component in a semisolid lesion. Such a solid component is seen as an indicator of more invasive behavior that warrants aggressive workup and resection. Ground glass lesions are followed by CT.

\section{Radiation Dose}

Low-dose CT is the technique used for screening. The only issue is that low dose is not well defined. In early studies, an effective dose $<5 \mathrm{mSv}$ was hailed as low dose. Presently, slim patients can be scanned with substantially less than $1 \mathrm{mSv}$ using modern scanners and iterative reconstruction techniques. As patients get more obese, a higher radiation dose is needed but this dose should rarely exceed $3 \mathrm{mSv}$ with modern technique. 
The radiation risk at high doses $>50 \mathrm{mSv}$ is well established from atomic bomb data and an increasing number of clinical studies. We know that in the age group screened ( $>50$ years), the risk for lung cancer induction is higher than the risk of induction of any other cancer. In the range between 5 and $50 \mathrm{mSv}$ only estimates can be made; usually a linear extrapolation to 0 is performed. Using this linear nonthreshold theory, the life-time attributable cancer risk at age 60 and exposure to $1 \mathrm{mSv}$ is calculated to be 1 in $20,000 .{ }^{24}$ It has to be noted, however, that no data exist below $5 \mathrm{mSv}$ because atomic bomb survivors exposed to less than this value were not monitored. The estimated radiation risk is so low that any other risk contaminates potential measurements.

\section{How to Organize a Screening Program?}

Given the positive recommendations by various professional societies, increasing numbers of lung cancer screening programs are being offered. The scope of the program has a strong influence on how it has to be organized. The issues to be tackled are: scanning protocol, reading protocol, follow-up protocol, and appropriate surgical management. Recruitment of participants will depend strongly on whether screening is offered as a one-time exam, as a local screening program or large-scale population screening.

CT scanning requires near-isotropic resolution for nodule volumetry, which means that CT sections of $1 \mathrm{~mm}$ or less have to be acquired. This can be achieved with any 16 -slice scanner. However, image quality will increase and radiation dose will decrease with more modern scanners. Automated exposure control for CT should be available. A low-dose setting should be chosen. Together with modern iterative reconstruction techniques, radiation dose can be kept below $1 \mathrm{mSv}$ for most patients and $<3 \mathrm{mSv}$ for the very obese. High-resolution filters should be avoided because they will cause excessive image noise and hamper evaluation of low-dose images. Radiographers can be trained to be able to scan more than 10 patients per hour using these protocols but good patient instructions before scanning are important to achieve this high throughput.

Evaluation of CT data requires searching for nodules and then characterizing and measuring these nodules. Detection is improved if not the original thin sections are used but processed images using maximum intensity projections or thin-slab volume rendering that make it easier to distinguish nodular structures from vessel cross-sections. ${ }^{25}$ Computeraided detection (CAD) tools improve the detection rate of radiologists but vary substantially with respect to sensitivity and false-positive rates. ${ }^{26}$ Computer-aided systems are also invaluable for automatically registering follow-up and baseline scans so that nodules can be found back more rapidly and evaluation can be sped up. Volumetry is superior to simple diameter measurements with respect to growth assessment. However, substantial variations between software exist, and always the same software should be used for follow-up. ${ }^{15}$ An essential part of reading is the creating of a report that provides nodule location and characteristics and provides suggestions for follow-up or further management. Creating this report can be the most time-consuming part of the process. Again, appropriate software tools speed up this process.

One-time screening or local screening programs can be based on radiologists reading the exams. When it comes to population screening, however, reading these scans becomes a challenge: although mammography screening can be performed in the order of minutes per participant, CT screening requires scrutinizing hundreds of images. This takes 10 to 15 minutes per case including documentation of findings. This limits the amount of participants that can be evaluated per radiologist to well below 50 per 8-hour working day. Even this number appears unrealistic because effects such as fatigue will limit this number even further. In the best of circumstances that means that one radiologist can serve around 10,000 participants per year. Given that a few million individuals need to be screened in the United States, a large number of dedicated readers will need to be made available. Those who have ever evaluated large numbers of screening scans themselves will know that screening is tedious and ultimately boring work that requires a high level of concentration. It is unlikely that enough radiologists are able and willing to do this kind of work, for which they are ultimately overqualified. For this reason, certified other personnel, such as radiographers, are considered for population screening. ${ }^{6}$ Again, large numbers of observers would have to be trained, and reading hours will have to be reduced to make the work bearable.

An alternative approach would come from improved CAD systems that could speed up evaluation if they could be used as a first reader, which means that only those lesions are further evaluated that are picked up by the system. Performance of CAD systems, however, varies extensively and the vast majority is incapable of such an approach. Modern systems that combine various CAD engines might, however, soon yield a performance good enough to consider this approach. ${ }^{26}$ Automated classification will probably be added in the coming years preparing the way for a fully automated analysis of screening scans, which would positively influence the cost-effectiveness of lung cancer screening. The main barriers are regulatory approval and good testing databases. In addition, just like radiologists, these systems will probably never be able to detect all cancers, which require adequate expectation management to avoid litigation.

One-time screening requires few special preparations because any potentially suspicious nodule will be sent to further workup via the health care system. Given that 20 to $30 \%$ of participants will require follow-up or further workup, this causes a major problem. In addition, new nodules detected at follow-up might trigger further scans in the future, which put even more stress on the insurers. Small or missed nodules that develop into cancers may become a cause of litigation. For this reason, local screening programs should be developed that include appropriate expectation management of participants and a regular follow-up scheme depending on the findings on the baseline scan. Since participants are followed at regular intervals, the risk of missing nodules is somewhat reduced because growing lesions will be seen at follow-up. 
Nodule management is still vastly based on expert opinion. ${ }^{22,27}$ New data on the probability of malignancy depending on nodule size and other factors ${ }^{21}$ as well as increasing knowledge about volume doubling times of malignant lesions ${ }^{19,23}$ will make it possible to create an approach based on probability of malignancy and optimum time to follow-up. An important factor to consider is appropriate workup of suspicious nodules. Percutaneous biopsy is frequently the only option for small peripheral solid nodules but direct videoassisted thoracoscopic surgery (VATS) resection may be considered in suspected T1a tumors. The role of bronchoscopy is being redefined as small lesions can often not be reached. ${ }^{6}$ PET scanning may have a role for detecting lymph nodes and metastases but its role in small primary tumors or subsolid lesions is limited by its detection threshold. For VATS resection, percutaneous markers should be considered as small or subsolid lesions are often hard to find. A dedicated team should be available that is familiar with the more advanced techniques required for workup and treatment.

\section{Expectation Management}

Expectations of individuals entering a screening program are probably unrealistic: a recent small study showed high expectation levels for accuracy of CT screening. ${ }^{28}$ It can be assumed that individuals entering a screening program expect to be reasonably well protected of dying from lung cancer. However, the $20 \%$ mortality reduction seen in $\mathrm{NLST}^{7}$ means that the majority of patients who will get lung cancer still will die from it: screening leads to a substantial reduction in the risk of dying from lung cancer but does not protect from it. Having participants understand this fact will be crucial for setting up an effective screening service without drowning in litigations. This is all the more important as the mortality of lung cancer is much higher than that of breast cancer, the other cancer that is screened for by imaging.

In addition, we know that not all potential cancers will be detected prospectively. We know from numerous studies that the detection rate of nodules varies with the scanning and reviewing technique used but also with the performance of the radiologist. ${ }^{25}$ The chance of missing a significant lesion is very small but it is nonzero. It increases as the nodules get smaller and are located closer to the lung hilum. This is a strong argument in favor of not only offering a one-time screening exam but a whole program that brings back screenings every 1 to 2 years and will be able to pick up missed lesions on baseline scans.

Anxiety management is another issue. Those who are screened can expect that a nodule will be found in many cases. Most of these are too small to warrant more rapid follow-up than the 1 to 2 years interval used in a screening program. Approximately 20 to $30 \%$ of cases will demonstrate an intermediate nodule that warrants more rapid follow-up. In the NLST, $27 \%$ of participants had a noncalcified nodules $\geq 4 \mathrm{~mm}$ that triggered further evaluation, of which only less than $4 \%$ had a lung cancer confirmed. ${ }^{7}$ A noncalcified nodule was found in $50 \%$ of participants in NELSON. In $19 \%$ of participants, an indeterminate nodule was seen that required follow-up. ${ }^{17}$ Less than $2 \%$ of participants in the baseline screening required immediate workup and $8 \%$ of the subjects with indeterminate nodules on baseline required workup after the 3-month follow-up scan. Slightly less than half of those who were referred for workup were diagnosed with lung cancer. This means that the vast majority of screeningdetected nodules will prove benign. It therefore has to be understood by a screening participant that the detection of a small or intermediate nodule is nothing special apart from the fact that is warrants closer survey.

A participant with high-risk nodule that requires referral will enter the health care system and become a patient who will have to undergo relatively risky procedures such as biopsies or even resection, of which, on average, more than half will have been done for a benign process. Again, it appears reasonable to have screening participants know this in advance to avoid litigation.

The issue of incidental additional findings is not fully resolved. Many of these findings bear prognostic value (see below), others will need to unnecessary workup and extra cost and morbidity. If reading of screening scans is solely done by radiographers or other trained and certified nonradiologists, then at least informed consent must be gained that states that incidental findings will not be searched for. Whether this is legally or ethically acceptable, is a point of discussion.

\section{What Else Could Be Screened for?}

Lung cancer screening using low-dose $\mathrm{CT}$ is not only able to detect lung cancers but also a variety of other diseases that can readily be assessed on noncontrast chest $\mathrm{CT}^{29}$ These scans can detect coronary calcifications, emphysema, bronchial disease, aortic valve calcifications, thoracic aortic aneurysms, and osteoporosis, to name but a few.

Since the screening CT exams are not electrocardiographic synchronized, pulsation artifacts hamper the evaluation of the heart and the coronaries. These artifacts do not allow to rule out the presence of coronary calcifications but they do not hamper the demonstration and quantification of larger amounts of calcium. Such calcifications detected on lung cancer screening $\mathrm{CT}$ are a very strong predictor of cardiovascular events and all-cause mortality. In fact, short-term mortality from cardiovascular events is even higher than lung cancer mortality. Individuals with high coronary calcium scores derived from nongated screening scans were found to have an up to nine times higher all-cause mortality within 2 years than those in whom no calcium was detected. ${ }^{30}$ Cardiovascular preventive treatment of high-risk individuals identified by lung screening might therefore substantially increase the overall survival benefit of the screening procedure.

Lung screening scans can be used to identify individuals with early chronic obstructive pulmonary disease (COPD), ${ }^{31}$ and also allow for distinction between emphysema and airway-predominant COPD phenotypes. With advances in treatment, progression of disease might be 
slowed down, again increasing the benefit of screening. Aortic valve calcifications are a strong predictor of aortic valve stenosis. Again, appropriate measures could be initiated and should this diagnosis be confirmed by echocardiography? Similar presumptions can be made for other diseases such as aneurysms or osteoporosis. By introducing a combined screening effort, the benefit of chest screening might be improved at minimum extra cost, since only an additional evaluation of the additional findings has to be added. No hard data, however, are present about the potential benefits of such an approach.

\section{Overdiagnosis}

The diagnosis and resection of histologically proven lung cancer in low-dose CT screening does not necessarily translate into improved lung cancer mortality. Lung cancers show a broad variety of biological behaviors: some grow very rapidly and metastasize early; others grow so slowly that they do not affect the patients' life expectancy, the latter being referred to as "overdiagnosis." Data from earlier trials suggest that overdiagnosis might be a relevant problem in lung cancer screening. In the Mayo Lung Project after 6 years of screening, 143 lung cancers were detected in the interventional arm (chest radiographs and sputum analysis) as compared with 87 in the control arm. ${ }^{32}$ After a follow-up of 5 more years, 10 catch-up tumors were diagnosed in the control group. The excess of 46 lung cancers in the interventional arm was considered to reflect overdiagnosis and the risk of overdiagnosis was estimated to be as high as $51 \%$. Other investigators concluded that based on a volume doubling time of 400 days as a cutoff to discriminate overdiagnosed (indolent) cases from genuine cases, the proportion of overdiagnosed cases would be as low as $5 \%{ }^{33}$ Currently, there are, however, no accepted criteria to discriminate indolent tumors from potentially deadly ones. Although a volume doubling time of more than 400 days is generally considered to be a good indicator of benignancy, data from the NLST indicate that growth between two points in time is not sufficient to predict future growth. ${ }^{34}$ Some lung cancers appear not to grow just exponentially but demonstrate a fairly flat growth curve. Some tumors may even remain stable in size for a long time before showing an accelerated growth. Important strategies to reduce detection of indolent disease (or inconsequential disease) include reducing frequency of screening examinations, focusing on high-risk populations and raising the threshold for recall and biopsy. ${ }^{35}$

\section{Summary}

The success of the NLST has triggered various national societies to recommend lung cancer screening. However, potentially contradicting results seen in European trials warrant some caution and call for pooled analysis of all European lung cancer screening trials to obtain enough power to come to a well-founded conclusion. In the meantime, given the evidence from the US trial, lung cancer screening is likely to be implemented in practice.
This implementation is likely to be a challenge: one-time screening is easy to implement but has the drawback of a large percentage of follow-up scans that will be induced and that have to be paid for, usually by the health care system. In addition, there is the chance that small nodules that were not followed or missed on the screening CT ultimately develop into cancers. The risk for screening providers and cost for health insurers is therefore nonnegligible. Since the NLST was based on programs with at least three CT screening exams, screening should only be offered in the setting of an institutional or government screening program that offers controlled screening intervals and well-defined nodule management. Nodule management will probably move from expert opinion-based approaches to a more rational, risk-based approach. Population screening provided by a government organization is somewhat different and mainly requires cost-efficient use of resources: if a nation-wide program is installed, the number of trained professionals that will evaluate the screening $\mathrm{CT}$ data will become a challenge, and increasing amount of automation using CAD tools are likely to play a role in the future.

Finally, expectation management is of paramount importance for a success when starting a screening program. Participants must recognize that screening will reduce their risk of dying from lung cancer but that screening is not perfect: there is the risk that a screen-detected cancer will still kill, that a nodule will not be picked up initially, that a cancer will not be visible on CT until too late, or that a cancer will develop between screening rounds. They also must recognize that there will be a high rate of follow-up CT (in the range of approximately $25 \%$ ) because of indeterminate lesions, the vast majority of which will be benign. Participants must also realize that there is a small chance that they will be operated upon for lesions that turns out benign, and that they may rarely suffer from complications related to such procedures.

There are still open questions about appropriate selection of participants, management and follow-up protocols, treatment of small cancers, and automation. By increasing the scope of evaluation from merely lung cancer to all kinds of treatable diseases that can be seen on screening chest $\mathrm{CT}$, the cost-benefit ratio is likely to be positively influenced and overall survival benefit will increase. It is now time to start a few implementation trials worldwide to study how chest screening can be made a success.

\section{References}

1 Siegel R, Naishadham D, Jemal A. Cancer statistics, 2013. CA Cancer J Clin 2013;63(1):11-30

2 Lung Cancer Mortality Reduction Act. Available at: http://www. gpo.gov/fdsys/pkg/BILLS-112hr1394ih/pdf/BILLS-112hr1394ih.pdf

3 Vazquez M, Carter D, Brambilla E, et al; International Early Lung Cancer Action Program Investigators. Solitary and multiple resected adenocarcinomas after CT screening for lung cancer: histopathologic features and their prognostic implications. Lung Cancer 2009;64(2):148-154 
4 Frost JK, Ball WC Jr, Levin ML, et al. Early lung cancer detection: results of the initial (prevalence) radiologic and cytologic screening in the Johns Hopkins study. Am Rev Respir Dis 1984;130(4): 549-554

5 Oken MM, Hocking WG, Kvale PA, et al; PLCO Project Team. Screening by chest radiograph and lung cancer mortality: the Prostate, Lung, Colorectal, and Ovarian (PLCO) randomized trial. JAMA 2011;306(17):1865-1873

6 Field JK, Oudkerk M, Pedersen JH, Duffy SW. Prospects for population screening and diagnosis of lung cancer. Lancet 2013; 382(9893):732-741

7 Aberle DR, Adams AM, Berg CD, et al; National Lung Screening Trial Research Team. Reduced lung-cancer mortality with low-dose computed tomographic screening. N Engl J Med 2011;365(5):395-409

8 Bach PB, Mirkin JN, Oliver TK, et al. Benefits and harms of CT screening for lung cancer: a systematic review. JAMA 2012; 307(22):2418-2429

9 Saghir Z, Dirksen A, Ashraf H, et al. CT screening for lung cancer brings forward early disease. The randomised Danish Lung Cancer Screening Trial: status after five annual screening rounds with low-dose CT. Thorax 2012;67(4):296-301

10 Infante M, Cavuto S, Lutman FR, et al; DANTE Study Group. A randomized study of lung cancer screening with spiral computed tomography: three-year results from the DANTE trial. Am J Respir Crit Care Med 2009;180(5):445-453

11 Pastorino U, Rossi M, Rosato V, et al. Annual or biennial CT screening versus observation in heavy smokers: 5-year results of the MILD trial. Eur J Cancer Prev 2012;21(3):308-315

12 Hassanein M, Callison JC, Callaway-Lane C, Aldrich MC, Grogan EL, Massion PP. The state of molecular biomarkers for the early detection of lung cancer. Cancer Prev Res (Phila) 2012;5(8):992-1006

13 Jaklitsch MT, Jacobson FL, Austin JH, et al. The American Association for Thoracic Surgery guidelines for lung cancer screening using low-dose computed tomography scans for lung cancer survivors and other high-risk groups. J Thorac Cardiovasc Surg 2012;144(1):33-38

14 Henschke CI, McCauley DI, Yankelevitz DF, et al. Early Lung Cancer Action Project: overall design and findings from baseline screening. Lancet 1999;354(9173):99-105

15 de Hoop B, Gietema H, van Ginneken B, Zanen P, Groenewegen G, Prokop M. A comparison of six software packages for evaluation of solid lung nodules using semi-automated volumetry: what is the minimum increase in size to detect growth in repeated CT examinations. Eur Radiol 2009;19(4):800-808

$16 \mathrm{Xu} \mathrm{DM}$, Gietema H, de Koning $\mathrm{H}$, et al. Nodule management protocol of the NELSON randomised lung cancer screening trial. Lung Cancer 2006;54(2):177-184

17 van Klaveren RJ, Oudkerk M, Prokop M, et al. Management of lung nodules detected by volume CT scanning. N Engl J Med 2009; 361(23):2221-2229

$18 \mathrm{Xu}$ DM, van der Zaag-Loonen HJ, Oudkerk M, et al. Smooth or attached solid indeterminate nodules detected at baseline CT screening in the NELSON study: cancer risk during 1 . year of follow-up. Radiology 2009;250(1):264-272

19 Heuvelmans MA, Oudkerk M, de Bock GH, et al. Optimisation of volume-doubling time cutoff for fast-growing lung nodules in CT lung cancer screening reduces false-positive referrals. Eur Radiol 2013;23(7):1836-1845

20 de Hoop B, van Ginneken B, Gietema H, Prokop M. Pulmonary perifissural nodules on CT scans: rapid growth is not a predictor of malignancy. Radiology 2012;265(2):611-616

21 McWilliams A, Tammemagi MC, Mayo JR, et al. Probability of cancer in pulmonary nodules detected on first screening CT. N Engl J Med 2013;369(10):910-919

22 Naidich DP, Bankier AA, MacMahon $\mathrm{H}$, et al. Recommendations for the management of subsolid pulmonary nodules detected at CT: a statement from the Fleischner Society. Radiology 2013;266(1): 304-317

23 Henschke CI, Yankelevitz DF, Mirtcheva R, McGuinness G, McCauley D, Miettinen OS; ELCAP Group. CT screening for lung cancer: frequency and significance of part-solid and nonsolid nodules. AJR Am J Roentgenol 2002;178(5):1053-1057

24 Hall EJ, Brenner DJ. Cancer risks from diagnostic radiology. Br J Radiol 2008;81(965):362-378

25 Peloschek P, Sailer J, Weber M, Herold CJ, Prokop M, SchaeferProkop C. Pulmonary nodules: sensitivity of maximum intensity projection versus that of volume rendering of 3D multidetector CT data. Radiology 2007;243(2):561-569

26 van Ginneken B, Schaefer-Prokop CM, Prokop M. Computer-aided diagnosis: how to move from the laboratory to the clinic. Radiology 2011;261(3):719-732

27 National Comprehensive Cancer Network. NCCN Clinical Practice Guidelines in Oncology: Lung Cancer Screening. Available at: http://www.nccn.org/professionals/physician_gls/pdf/lung_screening.pdf

28 Sinicrope PS, Rabe KG, Brockman TA, et al. Perceptions of lung cancer risk and beliefs in screening accuracy of spiral computed tomography among high-risk lung cancer family members. Acad Radiol 2010;17(8):1012-1025

29 Mets OM, de Jong PA, Prokop M. Computed tomographic screening for lung cancer: an opportunity to evaluate other diseases. JAMA 2012;308(14):1433-1434

30 Jacobs PC, Prokop M, van der Graaf Y, et al. Comparing coronary artery calcium and thoracic aorta calcium for prediction of allcause mortality and cardiovascular events on low-dose non-gated computed tomography in a high-risk population of heavy smokers. Atherosclerosis 2010;209(2):455-462

31 Mets OM, Buckens CF, Zanen P, et al. Identification of chronic obstructive pulmonary disease in lung cancer screening computed tomographic scans. JAMA 2011;306(16):1775-1781

32 Welch HG, Black WC. Overdiagnosis in cancer. J Natl Cancer Inst 2010;102(9):605-613

33 Yankelevitz DF, Kostis WJ, Henschke CI, et al. Overdiagnosis in chest radiographic screening for lung carcinoma: frequency. Cancer 2003;97(5):1271-1275

34 Lindell RM, Hartman TE, Swensen SJ, Jett JR, Midthun DE, Mandrekar JN. 5-year lung cancer screening experience: growth curves of 18 lung cancers compared to histologic type, CT attenuation, stage, survival, and size. Chest 2009;136(6):1586-1595

35 Esserman LJ, Thompson IM Jr, Reid B. Overdiagnosis and overtreatment in cancer: an opportunity for improvement. JAMA 2013; 310(8):797-798 